\title{
The association between CYP1A1 genetic polymorphisms and coronary artery disease in the Uygur and Han of China
}

Jin-Guo Zou ${ }^{1,2+}$, Yi-Tong Ma ${ }^{1 *}$, Xiang Xie ${ }^{1,2+}$, Yi-Ning Yang ${ }^{1}$, Shuo Pan ${ }^{1,2}$, Dilare Adi ${ }^{1,2}$, Fen Liu ${ }^{2}$ and Bang-Dang Chen ${ }^{2}$

\begin{abstract}
Background: The cytochrome P450, family 1, subfamily A, polypeptide 1 (CYP1A1) gene is expressed in the vascular endothelium, which metabolizes arachidonic acid into 20-hydroxyeicosatetraenoic acid (20-HETE) and epoxyeicosatrienoic acids (EETs). 20-HETE mediates cardiovascular homeostasis and growth response in vascular smooth muscle cells (VSMCs) as well as the anti-platelet effect. EETs are potent endogenous vasodilators and inhibitors of vascular inflammation. This study assessed the association between human CYP1A1 gene polymorphisms and coronary artery disease (CAD) in the Uygur and Han in China.
\end{abstract}

Methods: Two independent case-control studies that recruited Han (389 patients with CAD and 411 controls) and Uygur participants (293 patients with CAD and 408 controls) analyzed the relationship between CYP1A1 single nucleotide polymorphisms (SNPs: rs4886605, rs12441817, rs4646422 and rs1048943) and CAD. All patients with CAD and controls were genotyped for the four SNPs of CYP1A1 using TaqMan SNP genotyping assays.

Results: In the Uygur group, the distribution of the dominant model(CC vs $C T+\Pi$ ) of rs 4886605 for the total sample and the males was significantly different between CAD patients and control participants $(P=0.001$ and $P=0.012$, respectively), The difference remained significant after a multivariate adjustment $(P=0.018, P=0.015$, respectively). The rs 12441817 was also associated with $C A D$ in a dominant model for all participants $(P=0.003)$ and men $(P=0.012)$, and the difference remained significant after a multivariate adjustment $(P=0.016, P=0.002$, respectively). However, we did not observe differences in the Uygur females and Han group with regard to the allele frequency or genotypic distribution of rs4886605 and rs 12441817 between patients with CAD and control participants. Patients with CAD did not significantly differ from the control participants with regard to the distributions of rs4646422 and rs1048943 genotypes, the dominant model, the recessive model, or allele frequency in the Han and Uygur groups.

Conclusion: Both rs4886605 and rs12441817 SNPs of the CYP1A1 gene are associated with CAD in the Uygur population of China.

Keywords: CYPIA1, Single nucleotide polymorphism, Coronary artery disease, Case-control study

\footnotetext{
*Correspondence: myt_xj@sina.com

${ }^{\dagger}$ Equal contributors

${ }^{1}$ Department of Cardiology, First Affiliated Hospital of Xinjiang Medical

University, Urumqi 830054, People's Republic of China

Full list of author information is available at the end of the article
} 


\section{Introduction}

Coronary artery disease (CAD) accounts for nearly 40\% of all the Causes of mortality in developed countries $[1,2]$. CAD is a complex, multifactorial and polygenic disorder thought to result from the interaction between an individual's genetic makeup and various environmental factors [3]. Various gene variants are associated with CAD $[4,5]$.

Cytochrome P450 (CYP) is a super-family of cysteinoheme enzymes that mediate the oxidative metabolism of exogenous and endogenous molecules [6]. CYP enzymes play an important role in maintaining cardiovascular health because they catalyze the formation and/or metabolism of several endogenous molecules, such as cholesterol, androgens, estrogens, and several arachidonic acid metabolites, that affect several cardiovascular functions $[7,8]$. Mounting evidence demonstrates that CYP enzymes are involved in the pathogenesis of CAD $[9,10]$. Polymorphisms of CYP genes such as, CYP2C8, CYP2C9, CYP2J2 (epoxyeicosatrienoic acid[EET] synthesis) [11-13], CYP8A (prostacyclin synthesis) [14], CYP11B2 (aldosterone synthesis) [15], CYP17, and CYP19 (sex hormone synthesis) are related to CAD [16]. The human gene cytochrome P450, family 1 , subfamily A, polypeptide 1 (CYP1A1) is expressed in the vascular endothelium. In addition to the roles that CYP1A1 plays in metabolizing exogenous compounds such as polycyclic aromatic hydrocarbons (PAHs) and aromatic amines that increase the development of atherosclerotic lesions, it can metabolize arachidonic acid to terminal 20-hydroxyeicosatetraenoic acid (20-HETEs; 75-90\%) and, to a lesser extent, epoxyeicosatrienoic acids (EETs; 5-7\%) [17-19]. 20-HETE plays critical roles in the regulation of cardiovascular, renal and pulmonary homeostasis as well as the growth response in vascular smooth muscle cells (VSMCs), cardiac function and vascular tone [7]. In addition, 20-HETE has beneficial anti-platelet effects and inhibits sodium reabsorption in the renal tubules [20]. EETs generally have cardioprotective effects [1].

Recently, several reports investigated the association between CYP1A1 genetic polymorphisms and the risk of CAD associated with cigarette smoking. Wang et al. [9], Manfredi et al. [21], and Cornelis et al. [10], studied the association between the CYP1A1 MspI polymorphism, cigarette smoking, and the risk of CAD. They provided inconsistent results. According to the physiological and pathological mechanism of CYP1A1, the interaction between CYP1A1 and smoking can cause atherosclerosis. furthermore, this gene can metabolize arachidonic acid into 20-HETE and EETs, which can directly lead to atherosclerosis [1]; However, few studies have excluded the factors related to smoking and independently assessed the relationship between CYP1A1 and coronary heart disease.
The present case-control study aimed to assess the association between the human gene CYP1A1 and CAD excluding the risk factors related to smoking in the Uygur and Han population who are two major ethnic groups in Xinjiang, China. It can be further clarified physiological and pathological mechanism that CYP1A1 can cause CAD through Arachidonic acid metabolites such as 20HETE and EETs.

\section{Materials and methods}

\section{Ethical approval of the study protocol}

Written informed consent was obtained from all participants, who explicitly provided permission for all DNA analyses and the collection of relevant clinical data. The Ethics Committee of the First Affiliated Hospital of Xinjiang Medical University (Urumqi, China) approved this study, which was conducted according to the standards of the Declaration of Helsinki.

\section{Participants}

The participants were recruited from the Han and Uygur population who live in the Xinjiang Uygur Autonomous Region of China. All patients and controls received differential diagnoses for chest pain at the Cardiac Catheterization Laboratory of the First Affiliated Hospital of Xinjiang Medical University between 2006 and 2013. Highly skilled physicians performed all coronary angiography procedures using the Judkins approach [22]. At least two experienced imaging specialists interpreted the coronary angiography findings, and the final CAD diagnosis was made based on the angiography report.

We randomly sampled 293 Uygur patients with CAD and 408 ethnically and geographically matched participants for the control group. In addition, we randomly sampled 389 Han patients with CAD and 411 ethnically and geographically matched participants for the control group. CAD was defined as the presence of at least one significant coronary artery stenosis of more than $50 \%$ luminal diameter according to coronary angiography for all groups. All control participants also underwent a coronary angiogram and did not have coronary artery stenoses or show clinical or electrocardiogram evidence of myocardial infarction (MI) or CAD. Control participants were not healthy individuals; some were diagnosed with hypertension, DM or hyperlipidemia. Thus, the control group was exposed to the same risk factors of CAD; however, their coronary angiogram results were normal. Data and information regarding traditional coronary risk factors including hypertension, diabetes mellitus (DM), and smoking were collected from all study participants. The diagnosis of hypertension was established if patients were on antihypertensive medication or if the mean of 3 measurements of systolic blood pressure (SBP) was $>140 \mathrm{~mm} \mathrm{Hg}$ or diastolic blood pressure (DBP) $>90 \mathrm{~mm} \mathrm{Hg}$, respectively. 
Diabetes mellitus was diagnosed using the criteria of the World Health Organization (WHO). In addition, individuals with a fasting plasma glucose of $>7.0 \mathrm{mmol} / \mathrm{L}$, or with a history of diabetes or treatment with insulin were considered as diabetic. The smoking variable was dichotomized as smokers (including current and ex-smokers) and nonsmokers. All patients with impaired renal function, malignancy, connective tissue disease, valvular disease or chronic inflammatory disease were excluded.

\section{Blood collection and DNA extraction}

Fasting blood samples drawn via venipuncture in the catheter room were taken from all participants before cardiac catheterization. The blood samples were drawn into a 5-mL ethylene diamine tetraacetic acid (EDTA) tube and centrifuged at $4000 \times \mathrm{g}$ for $5 \mathrm{~min}$ to separate the plasma content. Genomic DNA was extracted from the peripheral leukocytes using the standard phenol-chloroform method. The DNA samples were stored at $-80^{\circ} \mathrm{C}$ until use. For use, the DNA was diluted to a concentration of $50 \mathrm{ng} / \mu \mathrm{L}$.

\section{Genotyping}

CYP1A1 is located on chromosome 15q24. This gene consists of approximately 6.068 kilobase pairs (kbp) and contains seven exons that are separated by six introns (Figure 1). A total of 287 SNPs are listed for the human
CYP1A1 gene in the National Center for Biotechnology Information SNP database (http://www.ncbi.nlm.nih.gov/ SNP). Using Haploview 4.2 and the HapMap phase II database, we obtained four tag SNPs (rs4886605, rs12441817, rs4646422 and rs1048943) using minor allele frequency (MAF) of $>0.1$ and linkage disequilibrium patterns with $\mathrm{r}^{2} \geq 0.5$ as a cut off. The position of SNP1, SNP2, SNP3 and SNP4 (rs4886605, rs12441817, rs4646422 and rs1048943) occurred in order of decreasing distance from the CYP1A1 gene 3'end (Figure 1). SNP1 (rs4886605) was located 8.037 kbp upstream from the start codon in exon 1; SNP2 (rs12441817) was located $7.863 \mathrm{kbp}$ upstream from the start codon in exon 1; SNP3 (rs4646422) was observed in the exon 2 region of the gene; and SNP4 (rs1048943) was observed in the exon 7 region of the gene. Genotyping was undertaken using the TaqMan ${ }^{\bullet}$ SNP Genotyping Assay (Applied Biosystems). The primers and probes used for the TaqMan ${ }^{\circ}$ SNP Genotyping Assays (ABI) were chosen based on information on ABI's website (http://appliedbiosystems. com.cn/). Thermal cycling was conducted using the Applied Biosystems 7900HT Standard Real-Time PCR System. Plates were read using the Sequence Detection Systems (SDS) automation controller software v2.4 (ABI). PCR amplification was performed using $2.5 \mu \mathrm{L}$ of TaqMan Universa Master Mix, $0.15 \mu \mathrm{L}$ probes and $1.85 \mathrm{ddH} 2 \mathrm{O}$ in a 6$\mu \mathrm{L}$ final reaction volume containing $1 \mu \mathrm{L}$ DNA. Thermal

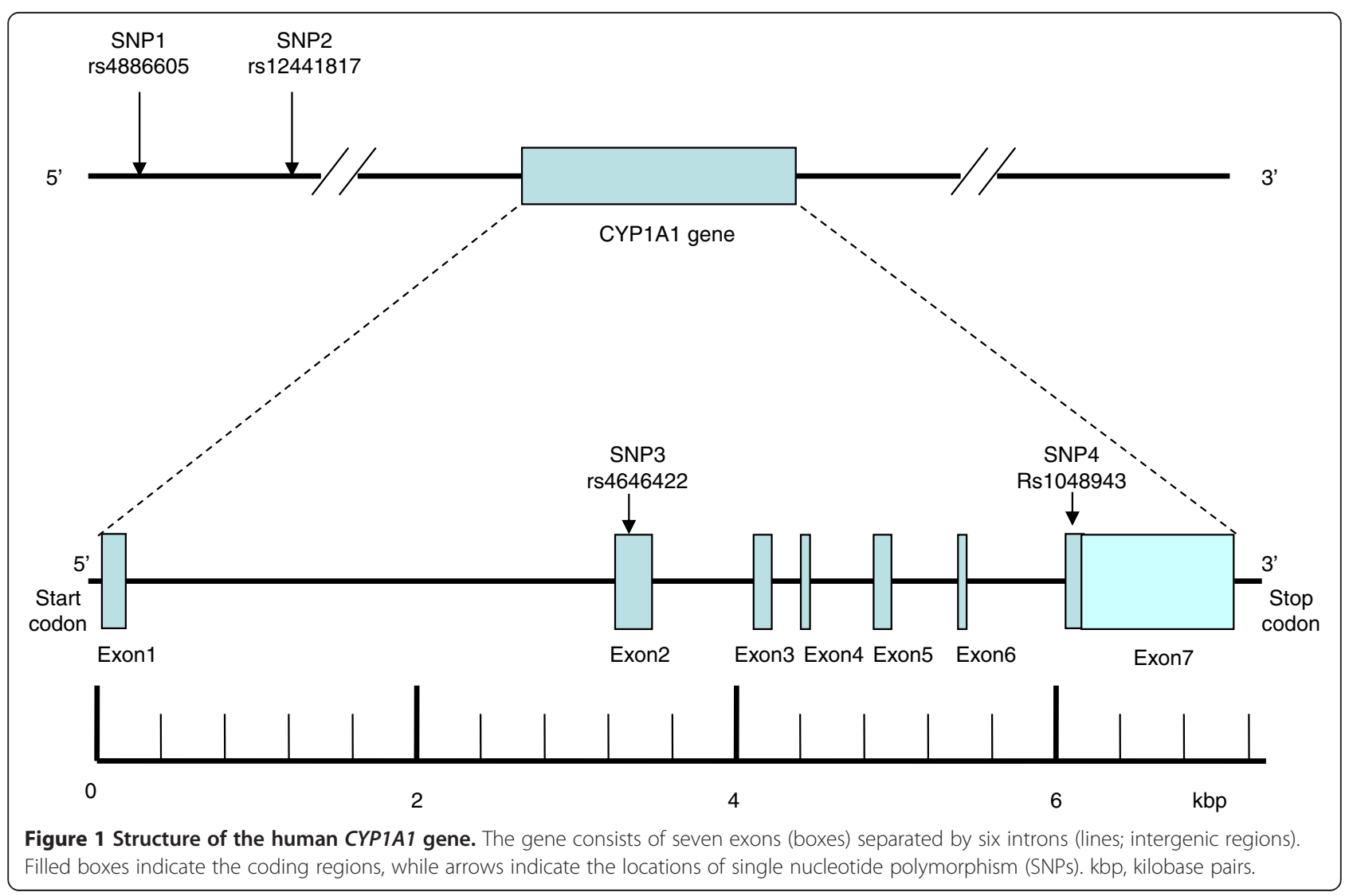


cycling conditions were as follows: $95^{\circ} \mathrm{C}$ for $5 \mathrm{~min}$; 40 cycles of $95^{\circ} \mathrm{C}$ for $15 \mathrm{~s}$; and $60^{\circ} \mathrm{C}$ for $1 \mathrm{~min}$. All 96-well plates were read using Sequence Detection Systems (SDS) automation controller software v2.4 (ABI).

\section{Biochemical analyses}

Serum concentrations of total cholesterol (TC), triglyceride (TG), glucose (Glu), high-density lipoprotein cholesterol (HDL-C), and low-density lipoprotein cholesterol (LDL-C), were measured using standard methods at the Central Laboratory of the First Affiliated Hospital of Xin jiang Medical University.

\section{Statistical analyses}

All continuous variables (e.g., age, BMI, pulse, and cholesterol levels) are presented as the means \pm standard deviation (S.D.). The differences between the CAD and control groups were analyzed using independent-samples $T$-test. The differences with regard to the frequencies of smoking, hypertension, diabetes mellitus, and CYP1A1 genotypes were analyzed using $X^{2}$ test or Fisher's exact test where appropriate. The Hardy-Weinberg equilibrium was assessed using a $X^{2}$ analysis. Logistic regression analyses with effect ratios (odds ratios [ORs] and 95\% CIs) were used to assess the contribution of the major risk factors. All statistical analyses were performed using SPSS 16.0 for Windows (SPSS Institute, Chicago, USA). P-value is 2-tailed, and Pvalues of less than 0.05 were considered significant.

\section{Results}

Table 1 shows the clinical characteristics of the Han and Uygur study participants. for total,men and women, The plasma concentrations of Glu and LDL-C as well as the rates of $\mathrm{EH}, \mathrm{DM}$, and smoking were all significantly higher for patients with CAD than control participants in the two ethnic population, and the plasma concentrations of Glu was significantly higher only for total and men group. BMI was significantly higher among total, men and women patients with CAD than their control counterparts in Uygur populations, and the plasma concentration of TC was significantly higher for women group.

Tables 2 and 3 show the distribution of the genotypes and alleles for the four CYP1A1 gene SNPs in the Han and Uygur populations. The genotypic distribution of each SNP matched the predicted Hardy-Weinberg equilibrium values for both ethnicities ( $P>0.05$ for the CAD and control groups; data not shown).

The distribution of the SNP1 (rs4886605) genotypes $(\mathrm{P}=0.004)$ and the additive model $(\mathrm{P}=0.027)$ significantly differed between the CAD and control participants in the entire Uygur sample. The distribution of SNP1 (rs4886605) alleles and the dominant model (CC vs $\mathrm{CT}+\mathrm{TT}$ ) significantly differed between $\mathrm{CAD}$ and control participants (alleles: $\mathrm{P}=0.006$ [total sample] and
$\mathrm{P}=0.021$ [men]; dominant model: $\mathrm{P}=0.001$ [total sample] and $\mathrm{P}=0.012$ [men]). The $\mathrm{T}$ allele of rs4886605 was found significantly more frequently in patients with CAD than controls (total: $46.76 \%$ vs $39.09 \%$; men: $46.43 \%$ vs $38.54 \%$ ). The dominant model (CC vs CT + TT) of rs4886605 was significantly lower among patients CAD than controls (total: $25.60 \%$ vs $37.50 \%$; men: $24.80 \%$ vs $36.10 \%)$. The distribution of SNP2 (rs12441817) genotypes $(\mathrm{P}=0.010)$ and the additive model $(\mathrm{P}=0.048)$ significantly differed between patients with CAD and controls for the entire sample. The distribution of SNP2 (rs12441817) alleles and the dominant model (TT vs CC + CT) significantly differed between patients $\mathrm{CAD}$ and controls (alleles: $\mathrm{P}=0.004$ [total sample] and $\mathrm{P}=0.013$ [men]; dominant model: $\mathrm{P}=0.003$ [total sample] and $\mathrm{P}=0.012$ [men]). The C allele of rs12441817 was found significantly more frequently in patients with CAD than control participants (total: $40.27 \%$ vs $32.84 \%$; men: $40.48 \%$ vs $32.20 \%$ ). The dominant model (TT vs CC + CT) of rs12441817 was significantly lower among patients with CAD than control participants (total: $34.10 \%$ vs $45.30 \%$; men: $33.30 \%$ vs $45.40 \%$ ). Moreover, significant differences were not observed between patients with CAD and control participants (for total participants, males or females) in the Uygur group with regard to the distributions of rs4646422 or rs1048943, the dominant model, the recessive model, or allele frequency $(P>0.05)$. Similarly, significant differences were not observed between patients with CAD and control participants (for total participants, males or females) within the Han group with regard to the distributions of rs4886605, rs12441817, rs4646422 or rs1048943, the dominant model, the recessive model, or allele frequency $(\mathrm{P}>0.05)$.

Tables 4 and 5 show that multiple logistic regression analyses were performed with TG, TC, HDL, Glu, LDL-C, EH, $\mathrm{DM}$, and smoking because these variables are the major confounding factors for CAD. Table 4: The significant difference observed with regard to rs4886605 was retained after adjustment for TG, TC, HDL, Glu, LDL-C, EH, DM, and smoking within the Uygur population (total participants: $\mathrm{OR}=0.368,95 \%$ confidence intervals $[\mathrm{CI}]=0.185-$ 0.530, $\mathrm{P}=0.018$; men: $\mathrm{OR}=0.350,95 \% \mathrm{CI}: 0.235-0.568$, $\mathrm{P}=0.015)$. Table 5: The significant difference observed with regard to rs12441817 was retained after multivariate adjustment for TG, TC, HDL, Glu, LDL-C, EH, DM, and smoking within Uygur population (total participants: $\mathrm{OR}=0.253$, 95\% confidence intervals $[\mathrm{CI}]=0.231-0.546, \quad \mathrm{P}=0.016$; men: $\mathrm{OR}=0.241,95 \% \mathrm{CI}=0.132-0.478, \mathrm{P}=0.002$ ).

\section{Discussion}

We found that variation in CYP1A1 gene is associated with $\mathrm{CAD}$ in the Uygur population of China. After multivariate adjustment, the associations between CYP1A1 gene polymorphisms with CAD were not modified. Our study is the 
Table 1 Han and Uygur population characteristics of study participants

\begin{tabular}{|c|c|c|c|c|c|c|c|c|c|c|c|c|c|c|c|c|c|c|}
\hline & \multicolumn{9}{|c|}{ Han } & \multicolumn{9}{|c|}{ Uygur } \\
\hline & \multicolumn{3}{|c|}{ Total } & \multicolumn{3}{|c|}{ Men } & \multicolumn{3}{|c|}{ Women } & \multicolumn{3}{|c|}{ Total } & \multicolumn{3}{|c|}{ Men } & \multicolumn{3}{|c|}{ Women } \\
\hline & CAD & Control & $P$ value & CAD & Control & $P$ value & CAD & Control & $P$ value & CAD & Control & $P$ value & CAD & Control & $P$ value & CAD & Control & $P$ value \\
\hline Number (n) & 389 & 411 & & 294 & 224 & & 95 & 187 & & 293 & 408 & & 210 & 205 & & 83 & 203 & \\
\hline Age (years) & $\begin{array}{c}58.73 \pm \\
7.32\end{array}$ & $\begin{array}{c}57.82 \pm \\
8.53\end{array}$ & 0.502 & $\begin{array}{c}59.82 \pm \\
8.24\end{array}$ & $\begin{array}{c}58.52 \pm \\
8.43\end{array}$ & 0.332 & $\begin{array}{c}58.21 \pm \\
6.37\end{array}$ & $\begin{array}{c}59.76 \pm \\
6.76\end{array}$ & 0.052 & $\begin{array}{c}56.97 \pm \\
5.58\end{array}$ & $\begin{array}{c}55.93 \pm \\
5.59\end{array}$ & 0.086 & $\begin{array}{c}56.57 \pm \\
8.33\end{array}$ & $\begin{array}{c}54.96 \pm \\
9.06\end{array}$ & 0.09 & $\begin{array}{c}54.20 \pm \\
3.89\end{array}$ & $\begin{array}{c}52.30 \pm \\
4.83\end{array}$ & 0.076 \\
\hline $\begin{array}{l}\text { BMl } \\
(\mathrm{kg} / \mathrm{m} 2)\end{array}$ & $\begin{array}{c}27.62 \pm \\
3.71\end{array}$ & $\begin{array}{c}28.23 \pm \\
2.74\end{array}$ & 0.428 & $\begin{array}{c}26.63 \pm \\
1.72\end{array}$ & $\begin{array}{c}26.34 \pm \\
1.75\end{array}$ & 0.201 & $\begin{array}{c}30.63 \pm \\
1.78\end{array}$ & $\begin{array}{c}30.17 \pm \\
2.23\end{array}$ & 0.069 & $\begin{array}{c}32.34 \pm \\
7.20\end{array}$ & $\begin{array}{c}28.11 \pm \\
4.37\end{array}$ & $<0.001^{*}$ & $\begin{array}{c}31.45 \pm \\
6.84\end{array}$ & $\begin{array}{c}28.48 \pm \\
3.98\end{array}$ & $<0.001^{*}$ & $\begin{array}{c}32.97 \pm \\
7.40\end{array}$ & $\begin{array}{c}26.73 \pm \\
5.41\end{array}$ & $<0.001^{*}$ \\
\hline $\begin{array}{l}\text { Pulse } \\
\text { (beats/min) }\end{array}$ & $\begin{array}{c}73.82 \pm \\
10.23\end{array}$ & $\begin{array}{c}73.13 \pm \\
10.01\end{array}$ & 0.673 & $\begin{array}{c}74.24 \pm \\
10.52\end{array}$ & $\begin{array}{c}73.76 \pm \\
10.23\end{array}$ & 0.324 & $\begin{array}{c}73.13 \pm \\
9.24\end{array}$ & $\begin{array}{c}72.45 \pm \\
8.53\end{array}$ & 0.802 & $\begin{array}{c}74.59 \pm \\
10.92\end{array}$ & $\begin{array}{c}76.56 \pm \\
7.93\end{array}$ & 0.38 & $\begin{array}{c}73.49 \pm \\
11.20\end{array}$ & $\begin{array}{c}74.76 \pm \\
8.45\end{array}$ & 0.732 & $\begin{array}{c}76.91 \pm \\
9.74\end{array}$ & $\begin{array}{c}80.38 \pm \\
5.17\end{array}$ & 0.339 \\
\hline $\begin{array}{l}\text { Glu } \\
(\mathrm{mmol} / \mathrm{L})\end{array}$ & $\begin{array}{c}6.12 \pm \\
2.34\end{array}$ & $\begin{array}{c}5.28 \pm \\
1.67\end{array}$ & $<0.001^{*}$ & $\begin{array}{c}5.63 \pm \\
1.68\end{array}$ & $\begin{array}{c}5.42 \pm \\
1.45\end{array}$ & $0.036^{*}$ & $\begin{array}{c}6.48 \pm \\
3.12\end{array}$ & $\begin{array}{c}5.42 \pm \\
1.59\end{array}$ & $<0.001^{*}$ & $\begin{array}{c}6.60 \pm \\
2.78\end{array}$ & $\begin{array}{c}5.24 \pm \\
2.38\end{array}$ & $<0.001^{*}$ & $\begin{array}{c}6.21 \pm \\
2.64\end{array}$ & $\begin{array}{c}5.20 \pm \\
2.55\end{array}$ & $<0.001^{*}$ & $\begin{array}{c}7.31 \pm \\
3.25\end{array}$ & $\begin{array}{c}5.37 \pm \\
2.41\end{array}$ & $<0.001^{*}$ \\
\hline $\begin{array}{l}\text { TG } \\
(\mathrm{mmol} / \mathrm{L})\end{array}$ & $\begin{array}{c}1.97 \pm \\
1.22\end{array}$ & $\begin{array}{c}1.82 \pm \\
1.76\end{array}$ & 0.978 & $\begin{array}{c}1.74 \pm \\
0.87\end{array}$ & $\begin{array}{c}1.58 \pm \\
0.69\end{array}$ & 0.284 & $\begin{array}{c}2.23 \pm \\
1.64\end{array}$ & $\begin{array}{c}1.62 \pm \\
1.18\end{array}$ & 0.254 & $\begin{array}{c}1.83 \pm \\
1.25\end{array}$ & $\begin{array}{c}1.76 \pm \\
1.35\end{array}$ & 0.435 & $\begin{array}{c}1.77 \pm \\
0.99\end{array}$ & $\begin{array}{c}1.83 \pm \\
1.50\end{array}$ & 0.638 & $\begin{array}{c}2.10 \pm \\
2.07\end{array}$ & $\begin{array}{c}1.70 \pm \\
1.24\end{array}$ & 0.061 \\
\hline $\begin{array}{l}\text { TC } \\
(\mathrm{mmol} / \mathrm{L})\end{array}$ & $\begin{array}{c}4.35 \pm \\
1.08\end{array}$ & $\begin{array}{c}4.12 \pm \\
0.74\end{array}$ & 0.262 & $\begin{array}{c}4.35 \pm \\
1.17\end{array}$ & $\begin{array}{c}4.26 \pm \\
0.82\end{array}$ & 0.431 & $\begin{array}{c}4.62 \pm \\
1.23\end{array}$ & $\begin{array}{c}4.52 \pm \\
0.85\end{array}$ & 0.873 & $\begin{array}{c}4.24 \pm \\
1.08\end{array}$ & $\begin{array}{c}4.15 \pm \\
0.98\end{array}$ & 0.34 & $\begin{array}{c}4.18 \pm \\
1.06\end{array}$ & $\begin{array}{c}4.13 \pm \\
0.97\end{array}$ & 0.565 & $\begin{array}{c}4.50 \pm \\
1.15\end{array}$ & $\begin{array}{c}4.17 \pm \\
0.97\end{array}$ & $0.037^{*}$ \\
\hline $\begin{array}{l}\mathrm{HDL} \\
(\mathrm{mmol} / \mathrm{L})\end{array}$ & $\begin{array}{c}1.14 \pm \\
0.23\end{array}$ & $\begin{array}{c}1.26 \pm \\
0.31\end{array}$ & 0.054 & $\begin{array}{c}1.12 \pm \\
0.26\end{array}$ & $\begin{array}{c}1.19 \pm \\
0.47\end{array}$ & 0.672 & $\begin{array}{c}1.15 \pm \\
0.36\end{array}$ & $\begin{array}{c}1.33 \pm \\
0.24\end{array}$ & 0.528 & $\begin{array}{c}0.98 \pm \\
0.13\end{array}$ & $\begin{array}{c}1.01 \pm \\
0.12\end{array}$ & 0.142 & $\begin{array}{c}0.97 \pm \\
0.31\end{array}$ & $\begin{array}{c}0.98 \pm \\
0.25\end{array}$ & 0.738 & $\begin{array}{c}1.044 \pm \\
0.20\end{array}$ & $\begin{array}{c}1.04 \pm \\
0.10\end{array}$ & 0.702 \\
\hline $\begin{array}{l}\text { LDL } \\
\text { (mmol/L) }\end{array}$ & $\begin{array}{c}2.53 \pm \\
0.79\end{array}$ & $\begin{array}{c}2.16 \pm \\
0.85\end{array}$ & $<0.001^{*}$ & $\begin{array}{c}2.62 \pm \\
0.84\end{array}$ & $\begin{array}{c}2.56 \pm \\
0.68\end{array}$ & $0.005^{*}$ & $\begin{array}{c}2.58 \pm \\
1.02\end{array}$ & $\begin{array}{c}2.24 \pm \\
0.58\end{array}$ & $<0.001^{*}$ & $\begin{array}{c}2.86 \pm \\
0.86\end{array}$ & $\begin{array}{c}2.59 \pm \\
0.97\end{array}$ & $<0.001^{*}$ & $\begin{array}{c}2.90 \pm \\
0.84\end{array}$ & $\begin{array}{c}2.56 \pm \\
0.99\end{array}$ & $<0.001^{*}$ & $\begin{array}{c}2.84 \pm \\
0.87\end{array}$ & $\begin{array}{c}2.72 \pm \\
0.89\end{array}$ & 0.356 \\
\hline EH (\%) & 59.32 & 47.63 & $0.023^{*}$ & 62.31 & 43.34 & $<0.001^{*}$ & 57.27 & 51.22 & $0.031^{*}$ & 42.68 & 18.42 & $<0.001^{*}$ & 34.35 & 20.01 & $<0.001^{*}$ & 51 & 16.53 & $<0.001^{*}$ \\
\hline DM (\%) & 27.23 & 13.56 & $<0.001^{*}$ & 22.74 & 15.03 & $<0.001^{*}$ & 31.18 & 12.26 & $<0.001^{*}$ & 26.71 & 12.84 & $<0.001^{*}$ & 21.53 & 7.73 & $<0.001^{*}$ & 30.69 & 17.95 & $<0.001^{*}$ \\
\hline Smoke (\%) & 58.48 & 31.43 & $<0.001^{*}$ & 87.59 & 56.38 & $<0.001^{*}$ & 12.13 & 3.62 & $0.002^{*}$ & 33.15 & 17.97 & $<0.001^{*}$ & 63.73 & 34.9 & $<0.001^{*}$ & 5.63 & 1.23 & $0.023^{*}$ \\
\hline
\end{tabular}


Table 2 Genotype and allele distributions in the control subjects and patients with CAD in the Han population

\begin{tabular}{|c|c|c|c|c|c|c|c|c|c|}
\hline \multirow[b]{2}{*}{ Variants } & \multicolumn{3}{|c|}{ Total } & \multicolumn{3}{|c|}{ Men } & \multicolumn{3}{|c|}{ Women } \\
\hline & CAD n (\%) & Control n (\%) & $P$ Value & CAD n (\%) & Control n (\%) & $P$ Value & CAD n (\%) & Control n (\%) & P Value \\
\hline \multicolumn{10}{|c|}{ rs4886605(SNP1) } \\
\hline \multicolumn{10}{|c|}{ Genotyping } \\
\hline CC & $79(20.3 \%)$ & $96(23.4 \%)$ & & $58(19.7 \%)$ & $51(22.8 \%)$ & & $21(22.1 \%)$ & $45(24.1 \%)$ & \\
\hline CT & 190(48.8\%) & $200(48.7 \%)$ & & $146(49.7 \%)$ & 119(53.1\%) & & $44(46.3 \%)$ & $81(43.3 \%)$ & \\
\hline$\pi$ & $120(30.8 \%)$ & 115(28.0\%) & 0.494 & $90(30.6 \%)$ & $54(24.1 \%)$ & 0.248 & $30(31.6 \%)$ & $61(32.6 \%)$ & 0.881 \\
\hline \multicolumn{10}{|c|}{ Dominant model } \\
\hline CC & $79(20.3 \%)$ & $96(23.4 \%)$ & & 58(19.7\%) & $51(22.8 \%)$ & & $21(22.1 \%)$ & $45(24.1 \%)$ & \\
\hline$\pi+C T$ & $310(79.7 \%)$ & $315(76.6 \%)$ & 0.297 & $236(80.3 \%)$ & 173(77.2\%) & 0.4 & 74(77.9\%) & $142(75.9 \%)$ & 0.713 \\
\hline \multicolumn{10}{|c|}{ Recessive model } \\
\hline$\pi$ & $120(30.8 \%)$ & $115(28.0 \%)$ & 0.494 & $90(30.6 \%)$ & $54(24.1 \%)$ & 0.248 & $30(31.6 \%)$ & $61(32.6 \%)$ & \\
\hline $\mathrm{CC}+\mathrm{CT}$ & $269(69.2 \%)$ & 296(72.0\%) & 0.373 & 204(69.4\%) & 170(75.9\%) & 0.102 & $65(68.4 \%)$ & $126(67.4 \%)$ & 0.86 \\
\hline \multicolumn{10}{|c|}{ Additive model } \\
\hline CT & 190(48.8\%) & $200(48.7 \%)$ & & $146(49.7 \%)$ & 119(53.1\%) & & $44(46.3 \%)$ & $81(43.3 \%)$ & \\
\hline$C C+\pi$ & 199(51.1\%) & $211(51.3 \%)$ & 0.959 & $148(50.3 \%)$ & 105(46.9\%) & 0.434 & $51(53.7 \%)$ & 106(56.7\%) & 0.632 \\
\hline \multicolumn{10}{|l|}{ Allele } \\
\hline C & $348(44.7 \%)$ & $392(47.7 \%)$ & & $262(44.6 \%)$ & $221(49.3 \%)$ & & $86(45.3 \%)$ & $171(45.7 \%)$ & \\
\hline T & $430(55.3 \%)$ & 430(52.3\%) & 0.236 & $326(55.4 \%)$ & $227(50.7 \%)$ & 0.127 & 104(54.7\%) & $203(54.3 \%)$ & 0.918 \\
\hline \multicolumn{10}{|c|}{ rs12441817(SNP2) } \\
\hline \multicolumn{10}{|c|}{ Genotyping } \\
\hline CC & $83(21.3 \%)$ & $83(20.2 \%)$ & & $65(22.1 \%)$ & $35(15.6 \%)$ & & 18(18.9\%) & 48(25.7\%) & \\
\hline$C T$ & $205(52.7 \%)$ & 199(48.4\%) & & $154(52.4 \%)$ & $121(54.0 \%)$ & & $51(53.7 \%)$ & 78(41.7\%) & \\
\hline$\pi$ & $101(26.0 \%)$ & 129(31.4\%) & 0.235 & $75(25.5 \%)$ & 68(30.4\%) & 0.141 & $26(27.4 \%)$ & $61(32.6 \%)$ & 0.153 \\
\hline \multicolumn{10}{|c|}{ Dominant model } \\
\hline$\pi$ & $101(26.0 \%)$ & 129(31.4\%) & 0.235 & $75(25.5 \%)$ & 68(30.4\%) & 0.141 & $26(27.4 \%)$ & $61(32.6 \%)$ & \\
\hline$C C+C T$ & 288(74.0\%) & 282(68.6\%) & 0.09 & 219(74.5\%) & 156(69.6\%) & 0.222 & $69(72.6 \%)$ & $126(67.4 \%)$ & 0.367 \\
\hline \multicolumn{10}{|c|}{ Recessive model } \\
\hline CC & $83(21.3 \%)$ & $83(20.2 \%)$ & & $65(22.1 \%)$ & $35(15.6 \%)$ & & 18(18.9\%) & $48(25.7 \%)$ & \\
\hline$T T+C T$ & $306(78.7 \%)$ & $328(79.8 \%)$ & 0.691 & $229(77.9 \%)$ & 189(84.4\%) & 0.064 & 77(81.1\%) & 139(74.3\%) & 0.208 \\
\hline \multicolumn{10}{|c|}{ Additive model } \\
\hline$C T$ & $205(52.7 \%)$ & 199(48.4\%) & & 154(52.4\%) & $121(54.0 \%)$ & & $51(53.7 \%)$ & 78(41.7\%) & \\
\hline$C C+\pi$ & 184(47.3\%) & $212(51.6 \%)$ & 0.226 & $140(47.6 \%)$ & 103(46.0\%) & 0.712 & $44(46.3 \%)$ & 109(58.3\%) & 0.056 \\
\hline \multicolumn{10}{|l|}{ Allele } \\
\hline C & $371(47.7 \%)$ & $365(44.4 \%)$ & & 284(48.3\%) & $191(42.6 \%)$ & & $87(45.8 \%)$ & $174(46.5 \%)$ & \\
\hline $\mathrm{T}$ & $407(52.3 \%)$ & $457(55.6 \%)$ & 0.188 & $304(51.7 \%)$ & $257(57.4 \%)$ & 0.07 & $103(54.2 \%)$ & $200(53.5 \%)$ & 0.869 \\
\hline \multicolumn{10}{|c|}{ rs4646422(SNP3) } \\
\hline \multicolumn{10}{|c|}{ Genotyping } \\
\hline AA & $270(69.4 \%)$ & 286(69.6\%) & & 206(70.1\%) & 152(67.9\%) & & $64(67.4 \%)$ & 134(71.7\%) & \\
\hline$A G$ & $102(26.2 \%)$ & $115(28.0 \%)$ & & $74(25.2 \%)$ & $67(29.9 \%)$ & & $28(29.5 \%)$ & $48(25.7 \%)$ & \\
\hline GG & 17(4.4\%) & $10(2.4 \%)$ & 0.294 & 14(4.8\%) & $5(2.2 \%)$ & 0.187 & $3(3.2 \%)$ & $5(2.7 \%)$ & 0.759 \\
\hline \multicolumn{10}{|c|}{ Dominant model } \\
\hline AA & $270(69.4 \%)$ & 286(69.6\%) & & 206(70.1\%) & 152(67.9\%) & & 64(67.4\%) & 134(71.7\%) & \\
\hline$G G+A G$ & 119(30.6\%) & 125(30.4\%) & 0.957 & $88(30.0 \%)$ & $72(32.1 \%)$ & 0.59 & $31(32.6 \%)$ & $53(28.4 \%)$ & 0.457 \\
\hline
\end{tabular}

Recessive model 
Table 2 Genotype and allele distributions in the control subjects and patients with CAD in the Han population (Continued)

\begin{tabular}{|c|c|c|c|c|c|c|c|c|c|}
\hline GG & $17(4.4 \%)$ & $10(2.4 \%)$ & 0.294 & $14(4.8 \%)$ & $5(2.2 \%)$ & 0.187 & $3(3.2 \%)$ & $5(2.7 \%)$ & \\
\hline$A A+A G$ & 372(95.6\%) & $401(97.6 \%)$ & 0.129 & $280(95.2 \%)$ & 219(97.8\%) & 0.129 & $92(96.8 \%)$ & 182(97.3\%) & 0.818 \\
\hline \multicolumn{10}{|c|}{ Additive model } \\
\hline AG & $102(26.2 \%)$ & $115(28.0 \%)$ & & $74(25.2 \%)$ & $67(29.9 \%)$ & & $28(29.5 \%)$ & $48(25.7 \%)$ & \\
\hline$A A+G G$ & 287(73.8\%) & $296(72.0 \%)$ & 0.576 & $220(74.8 \%)$ & 157(70.1\%) & 0.23 & $67(70.5 \%)$ & 139(74.3\%) & 0.496 \\
\hline \multicolumn{10}{|l|}{ Allele } \\
\hline A & $642(82.5 \%)$ & 687(83.6\%) & & $486(82.7 \%)$ & $371(82.8 \%)$ & & 156(82.1\%) & $316(84.5 \%)$ & \\
\hline G & 136(17.5\%) & 135(16.4\%) & 0.573 & 102(17.3\%) & $77(17.2 \%)$ & 0.946 & $34(17.9 \%)$ & $58(15.5 \%)$ & 0.468 \\
\hline \multicolumn{10}{|c|}{ rs1048943(SNP4) } \\
\hline \multicolumn{10}{|c|}{ Genotyping } \\
\hline CC & $20(5.1 \%)$ & $17(4.1 \%)$ & & 15(5.1\%) & $8(3.6 \%)$ & & $5(5.3 \%)$ & $9(4.8 \%)$ & \\
\hline$C T$ & 152(39.1\%) & 148(36.0\%) & & 115(39.1\%) & $80(35.7 \%)$ & & $37(38.9 \%)$ & 68(36.4\%) & \\
\hline$\pi$ & $217(55.8 \%)$ & $246(59.9 \%)$ & 0.47 & $164(55.8 \%)$ & $136(60.7 \%)$ & 0.45 & $53(55.8 \%)$ & $110(58.8 \%)$ & 0.888 \\
\hline \multicolumn{10}{|c|}{ Dominant model } \\
\hline$\pi$ & $217(55.8 \%)$ & 246(59.9\%) & & 164(55.8\%) & $136(60.7 \%)$ & & $53(55.8 \%)$ & $110(58.8 \%)$ & \\
\hline $\mathrm{CC}+\mathrm{CT}$ & 172(44.2\%) & $165(40.1 \%)$ & 0.244 & $130(44.2 \%)$ & 88(39.3\%) & 0.26 & $42(44.2 \%)$ & $77(41.2 \%)$ & 0.626 \\
\hline \multicolumn{10}{|c|}{ Recessive model } \\
\hline CC & $20(5.1 \%)$ & 17(4.1\%) & & 15(5.1\%) & $8(3.6 \%)$ & & $5(5.3 \%)$ & $9(4.8 \%)$ & \\
\hline$T T+C T$ & 369(94.9\%) & 394(95.9\%) & 0.499 & 279(94.9\%) & 216(96.4\%) & 0.402 & $90(94.7 \%)$ & 178(95.2\%) & 0.87 \\
\hline \multicolumn{10}{|c|}{ Additive model } \\
\hline$C T$ & 152(39.1\%) & 148(36.0\%) & & 115(39.1\%) & $80(35.7 \%)$ & & $37(38.9 \%)$ & 68(36.4\%) & \\
\hline$C C+\pi$ & $237(60.9 \%)$ & $263(64.0 \%)$ & 0.371 & 179(60.9\%) & $144(64.3 \%)$ & 0.429 & $58(61.1 \%)$ & $119(63.6 \%)$ & 0.671 \\
\hline \multicolumn{10}{|l|}{ Allele } \\
\hline C & $192(24.7 \%)$ & 182(22.1\%) & & $145(24.7 \%)$ & $96(21.4 \%)$ & & $47(24.7 \%)$ & $86(23.0 \%)$ & \\
\hline $\mathrm{T}$ & $586(75.3 \%)$ & $640(77.9 \%)$ & 0.231 & $443(75.3 \%)$ & $352(78.6 \%)$ & 0.223 & 143(75.3\%) & 288(77.0\%) & 0.645 \\
\hline
\end{tabular}

CAD, coronary artery disease; SNP, single-nucleotide polymorphism.

first case-control study to investigate the association between the human CYP1A1 gene and CAD in the Uygur and Han populations in western China.

Several CYP enzyme families have been identified in the heart, endothelium, and smooth muscle of blood vessels. A link between the expression and activity of CYP and cardiovascular diseases (CVDs) such as hypertension, CAD, heart failure, stroke, cardiomyopathy, and arrhythmia has been established [7]. CYP1A1 polymorphisms can affect the metabolism of arachidonic acid, thereby resulting in an altered generating capacity of 20-HETE and EETs . 20-HETE have 5 physiological functions. First, 20-HETE increases the intracellular calcium concentration and regulates the vasoconstrictor responses of angiotensin II, vasopressin, and norepinephrine [23-25]. Second, 20-HETE increases thymidine incorporation in cells and regulates growth responses in vascular smooth muscle cells (VSMCs) in response to norepinephrine and angiotensin II [26-28]. Third, 20-HETE inhibits $\mathrm{Na}+-\mathrm{K}+-$ ATPase activity and sodium transport in the proximal tubule and is a key mediator of the longterm control of arterial pressure [29-31]. Fourth, 20-HETE significantly contributes to ischemia -reperfusion injury, and the exogenous administration of 20-HETE significantly increases infarct size [32,33]. Fifth, 20-HETE inhibits platelet aggregation and the formation of thromboxane A2 (TxA2) during platelet activation [34]. in addition, CYP1A1 also metabolize arachidonic acid to EETs, which are potent endogenous vasodilators, inhibitors of vascular inflammation, and possessors of potent vasodilatory and antiapoptotic and anti-thrombotic properties in the cardiovascular system [35].

Several genetic polymorphisms have been reported in the CYP1A1 gene including $\mathrm{T} 6235 \mathrm{C}$ and $\mathrm{T} 5639 \mathrm{C}$ in the 3 '-flanking region, A4889G and C4887A at exon 7 [36]. Functional studies have revealed that these polymorphisms are associated with increased CYP1A1 activity and/or inducibility [37]. Wang et al. [9] reported significant association between CYP1A1 MspI polymorphism and the risk of coronary artery disease(CAD) in cigarette smoking. This increased risk was more evident among light smokers with OR of 3.44, but not observed in nonsmokers or heavy smokers. Nevertheless, Manfredi et al. 
Table 3 Genotype and allele distributions in control subjects and patients with CAD in the Uygur population

\begin{tabular}{|c|c|c|c|c|c|c|c|c|c|}
\hline \multirow[b]{2}{*}{ Variants } & \multicolumn{3}{|c|}{ Total } & \multicolumn{3}{|c|}{ Men } & \multicolumn{3}{|c|}{ Women } \\
\hline & CAD n (\%) & Control n (\%) & P Value & CAD n (\%) & Control n (\%) & $P$ Value & CAD n (\%) & Control n(\%) & P Value \\
\hline \multicolumn{10}{|c|}{ rs4886605(SNP1) } \\
\hline \multicolumn{10}{|c|}{ Genotyping } \\
\hline CC & $75(25.60 \%)$ & 153(37.50\%) & & $52(24.80 \%)$ & 74(36.10\%) & & $23(27.70 \%)$ & 79(38.90\%) & \\
\hline$C T$ & 162(55.30\%) & $191(46.80 \%)$ & & $121(57.60 \%)$ & 104(50.70\%) & & $41(49.40 \%)$ & $87(42.90 \%)$ & \\
\hline Tा & $56(19.10 \%)$ & $64(15.70 \%)$ & $0.004^{*}$ & $37(17.60 \%)$ & $27(13.20 \%)$ & $0.036^{*}$ & 19(22.90\%) & $37(18.20 \%)$ & 0.193 \\
\hline \multicolumn{10}{|c|}{ Dominant model } \\
\hline CC & $75(25.60 \%)$ & 153(37.50\%) & & $52(24.80 \%)$ & $74(36.10 \%)$ & & $23(27.70 \%)$ & 79(38.90\%) & \\
\hline$T T+C T$ & 218(74.40\%) & $255(62.50 \%)$ & $0.001^{*}$ & 158(75.20\%) & $131(63.90 \%)$ & $0.012^{*}$ & $60(72.30 \%)$ & $124(61.10 \%)$ & 0.073 \\
\hline \multicolumn{10}{|c|}{ Recessive model } \\
\hline Tा & $56(19.10 \%)$ & $64(15.70 \%)$ & & $37(17.60 \%)$ & $27(13.20 \%)$ & & $19(22.90 \%)$ & $37(18.20 \%)$ & \\
\hline $\mathrm{CC}+\mathrm{CT}$ & 237(80.90\%) & $344(84.30 \%)$ & 0.235 & $173(82.40 \%)$ & 178(86.80\%) & 0.21 & 64(77.10\%) & 166(81.80\%) & 0.367 \\
\hline \multicolumn{10}{|c|}{ Additive model } \\
\hline$C T$ & 162(55.30\%) & $191(46.80 \%)$ & & $121(57.60 \%)$ & 104(50.70\%) & & $41(49.40 \%)$ & $87(42.90 \%)$ & \\
\hline$C C+\pi$ & $131(44.70 \%)$ & $217(53.20 \%)$ & $0.027^{*}$ & $89(42.40 \%)$ & $101(49.30 \%)$ & 0.159 & $42(50.60 \%)$ & 116(57.10\%) & 0.313 \\
\hline \multicolumn{10}{|l|}{ Allele } \\
\hline C & 312(53.24\%) & $497(60.91 \%)$ & & $225(53.57 \%)$ & $252(61.46 \%)$ & & $87(52.41 \%)$ & $245(60.34 \%)$ & \\
\hline $\mathrm{T}$ & $274(46.76 \%)$ & 319(39.09\%) & $0.006^{*}$ & $195(46.43 \%)$ & 158(38.54\%) & $0.021^{*}$ & 79(47.59\%) & $161(39.66 \%)$ & 0.081 \\
\hline \multicolumn{10}{|c|}{ rs12441817(SNP2) } \\
\hline \multicolumn{10}{|c|}{ Genotyping } \\
\hline CC & $43(14.70 \%)$ & $45(11.00 \%)$ & & $30(14.30 \%)$ & $20(9.80 \%)$ & & $13(15.70 \%)$ & $25(12.30 \%)$ & \\
\hline$C T$ & 150(51.20\%) & $178(43.60 \%)$ & & $110(52.40 \%)$ & $92(44.90 \%)$ & & $40(48.20 \%)$ & $86(42.40 \%)$ & \\
\hline$\pi$ & 100(34.10\%) & 185(45.30\%) & $0.01^{*}$ & 70(33.30\%) & $93(45.40 \%)$ & $0.034^{*}$ & $30(36.10 \%)$ & $92(45.30 \%)$ & 0.348 \\
\hline \multicolumn{10}{|c|}{ Dominant model } \\
\hline$\Pi$ & 100(34.10\%) & 185(45.30\%) & & 70(33.30\%) & $93(45.40 \%)$ & & $30(36.10 \%)$ & $92(45.30 \%)$ & \\
\hline$C C+C T$ & 193(65.90\%) & $223(54.60 \%)$ & $0.003^{*}$ & $140(66.70 \%)$ & $112(54.60 \%)$ & $0.012^{*}$ & $53(63.90 \%)$ & $111(54.70 \%)$ & 0.154 \\
\hline \multicolumn{10}{|c|}{ Recessive model } \\
\hline CC & $43(14.70 \%)$ & $45(11.00 \%)$ & & $30(14.30 \%)$ & $20(9.80 \%)$ & & $13(15.70 \%)$ & $25(12.30 \%)$ & \\
\hline$T T+C T$ & 250(85.30\%) & $363(89.00 \%)$ & 0.151 & 180(85.70\%) & 185(90.20\%) & 0.156 & $70(84.30 \%)$ & 178(87.70\%) & 0.449 \\
\hline \multicolumn{10}{|c|}{ Additive model } \\
\hline$C T$ & 150(51.20\%) & 178(43.60\%) & & $110(52.40 \%)$ & $92(44.90 \%)$ & & $40(48.20 \%)$ & $86(42.40 \%)$ & \\
\hline$C C+\pi$ & 143(48.80\%) & $230(56.40 \%)$ & $0.048^{*}$ & $100(47.60 \%)$ & $113(55.10 \%)$ & 0.126 & $43(51.80 \%)$ & $117(57.60 \%)$ & 0.368 \\
\hline \multicolumn{10}{|l|}{ Allele } \\
\hline C & $236(40.27 \%)$ & 268(32.84\%) & & $170(40.48 \%)$ & 132(32.20\%) & & 66(39.78\%) & 136(33.50\%) & \\
\hline $\mathrm{T}$ & $350(59.73 \%)$ & $548(67.16 \%)$ & $0.004^{*}$ & $250(59.52 \%)$ & $278(67.8 \%)$ & $0.013^{*}$ & $100(60.22 \%$ & $270(66.5 \%)$ & 0.155 \\
\hline \multicolumn{10}{|c|}{ rs4646422(SNP3) } \\
\hline \multicolumn{10}{|c|}{ Genotyping } \\
\hline AA & 251(85.7\%) & $350(85.80 \%)$ & & $178(84.80 \%)$ & 177(86.30\%) & & 73(88.00\%) & 173(85.20\%) & \\
\hline AG & $39(13.30 \%)$ & $53(13.00 \%)$ & & $32(15.20 \%)$ & $26(12.70 \%)$ & & $7(8.40 \%)$ & $27(13.30 \%)$ & \\
\hline GG & $3(1.00 \%)$ & $5(1.20 \%)$ & 0.963 & $0(0.00 \%)$ & $2(1.00 \%)$ & 0.319 & $3(3.60 \%)$ & $3(1.50 \%)$ & 0.291 \\
\hline \multicolumn{10}{|c|}{ Dominant model } \\
\hline AA & $251(85.70 \%)$ & $350(85.80 \%)$ & & $178(84.80 \%)$ & $177(86.30 \%)$ & & 73(88.00\%) & 173(85.20\%) & \\
\hline $\mathrm{GG}+\mathrm{AG}$ & $42(14.30 \%)$ & $58(14.20 \%)$ & 0.965 & $32(15.20 \%)$ & $28(13.70 \%)$ & 0.647 & $10(12.00 \%)$ & $30(14.80 \%)$ & 0.546 \\
\hline
\end{tabular}

Recessive model 
Table 3 Genotype and allele distributions in control subjects and patients with CAD in the Uygur population (Continued)

\begin{tabular}{|c|c|c|c|c|c|c|c|c|c|}
\hline GG & $3(1.00 \%)$ & $5(1.20 \%)$ & & $0(0.00 \%)$ & $2(1.00 \%)$ & & $3(3.60 \%)$ & $3(1.50 \%)$ & \\
\hline$A A+A G$ & 290(99.00\%) & 403(98.80\%) & 0.803 & $210(100.00 \%)$ & 203(99.00\%) & 0.243 & $80(96.40 \%)$ & 200(98.50\%) & 0.275 \\
\hline \multicolumn{10}{|c|}{ Additive model } \\
\hline$A G$ & $39(13.30 \%)$ & $53(13.00 \%)$ & & $32(15.20 \%)$ & $26(12.70 \%)$ & & $7(8.40 \%)$ & 27(13.30\%) & \\
\hline$A A+G G$ & 254(86.70\%) & $355(87.00 \%)$ & 0.901 & 178(84.80\%) & 179(87.30\%) & 0.453 & 76(91.60\%) & 176(86.70\%) & 0.248 \\
\hline \multicolumn{10}{|l|}{ Allele } \\
\hline A & $541(92.32 \%)$ & 753(92.28\%) & & 388(92.38\%) & $380(92.68 \%)$ & & 153(92.17\%) & 373(91.87\%) & \\
\hline G & $45(7.68 \%)$ & 63(7.72\%) & 0.977 & $32(7.62 \%)$ & $30(7.31 \%)$ & 0.869 & 13(7.83\%) & $33(8.13 \%)$ & 0.906 \\
\hline \multicolumn{10}{|c|}{ rs1048943(SNP4) } \\
\hline \multicolumn{10}{|c|}{ Genotyping } \\
\hline CC & $5(1.70 \%)$ & $11(2.70 \%)$ & & $3(1.40 \%)$ & $5(2.40 \%)$ & & $2(2.40 \%)$ & $6(3.00 \%)$ & \\
\hline$C T$ & $85(29.00 \%)$ & $112(27.50 \%)$ & & $63(30.00 \%)$ & $54(26.30 \%)$ & & $22(26.50 \%)$ & $58(28.60 \%)$ & \\
\hline Tा & 203(69.30\%) & 285(69.90\%) & 0.642 & 144(68.60\%) & 146(71.20\%) & 0.562 & $59(71.10 \%)$ & 139(68.50\%) & 0.899 \\
\hline \multicolumn{10}{|c|}{ Dominant model } \\
\hline$\pi$ & 203(69.30\%) & 285(69.90\%) & & 144(68.60\%) & 146(71.20\%) & & $59(71.10 \%)$ & 139(68.50\%) & \\
\hline $\mathrm{CC}+\mathrm{CT}$ & $90(30.70 \%)$ & 123(30.20\%) & 0.872 & $66(31.40 \%)$ & $59(28.70 \%)$ & 0.557 & $24(28.90 \%)$ & $64(31.60 \%)$ & 0.664 \\
\hline \multicolumn{10}{|c|}{ Recessive model } \\
\hline CC & $5(1.70 \%)$ & $11(2.70 \%)$ & & $3(1.40 \%)$ & $5(2.40 \%)$ & & $2(2.40 \%)$ & $6(3.00 \%)$ & \\
\hline$T T+C T$ & 288(98.30\%) & 397(97.40\%) & 0.387 & 207(98.60\%) & 200(97.50\%) & 0.452 & $81(97.60 \%)$ & 197(97.10\%) & 0.797 \\
\hline \multicolumn{10}{|c|}{ Additive model } \\
\hline$C T$ & $85(29.00 \%)$ & $112(27.50 \%)$ & & 63(30.00\%) & $54(26.30 \%)$ & & $22(26.50 \%)$ & $58(28.60 \%)$ & \\
\hline$C C+\pi$ & 208(71.00\%) & $296(72.60 \%)$ & 0.651 & 147(70.00\%) & $151(73.60 \%)$ & 0.408 & $61(73.50 \%)$ & $145(71.50 \%)$ & 0.724 \\
\hline \multicolumn{10}{|l|}{ Allele } \\
\hline C & $95(16.21 \%)$ & 134(16.42\%) & & $69(16.43 \%)$ & $64(15.61 \%)$ & & $26(15.66 \%)$ & $70(17.24 \%)$ & \\
\hline T & $491(83.79 \%)$ & 682(83.58\%) & 0.916 & $351(83.57 \%)$ & $346(84.39 \%)$ & 0.748 & $140(84.33 \%)$ & 336(82.76\%) & 0.647 \\
\hline
\end{tabular}

CAD, coronary artery disease; SNP, single-nucleotide polymorphism; *P $<0.05$.

[21] found no significant association was observed between CYP1A1 MspI polymorphism and the presence of CAD, or the number of significantly diseased vessels in smokers. In agreement with this study, Cornelis et al. [10] reported no significant association between CYP1A1 polymorphism and the risk of myocardial infarction (MI) in a study from Costa Rica. Chih-Ching Yeh et al. [38] report CYP1A1*2C polymorphism, particularly in nonsmokers, may be associated with the individual susceptibility to CAD. From the above example, we have considered

Table 4 Multiple logistic regression analysis for CAD patients and control subjects of Uygur population (rs4886605)

\begin{tabular}{|c|c|c|c|c|c|c|c|c|c|}
\hline & \multicolumn{3}{|c|}{ Total } & \multicolumn{3}{|c|}{ Men } & \multicolumn{3}{|c|}{ Women } \\
\hline & OR & $95 \% \mathrm{Cl}$ & $\mathbf{P}$ & OR & $95 \% \mathrm{Cl}$ & $\mathbf{P}$ & OR & $95 \% \mathrm{Cl}$ & $\mathbf{P}$ \\
\hline Dominant model (CC vs CT + TT) & 0.368 & $0.185-0.530$ & 0.018 & 0.35 & $0.235-0.568$ & 0.015 & 0.626 & $0.321-1.412$ & 0.204 \\
\hline Smoking & 8.29 & $5.365-12.846$ & $<0.001$ & 10.254 & $5.713-18.375$ & $<0.001$ & 0.232 & $0.039-1.825$ & 0.262 \\
\hline $\mathrm{EH}$ & 6.651 & $3.957-10.952$ & $<0.001$ & 9.81 & $4.94-20.513$ & $<0.001$ & 4.103 & $1.875-9.184$ & $<0.001$ \\
\hline DM & 1.524 & $0.949-2.495$ & 0.284 & 1.902 & $0.926-3.854$ & 0.087 & 0.95 & $0.358-2.243$ & 0.757 \\
\hline Glu & 1.352 & $1.241-1.482$ & $<0.001$ & 1.348 & $1.183-1.517$ & $<0.001$ & 1.485 & $1.253-1.762$ & $<0.001$ \\
\hline TG & 1.347 & $1.273-1.638$ & 0.251 & 1.384 & $1.169-1.615$ & 0.015 & 1.653 & $1.321-1.859$ & 0.021 \\
\hline TC & 1.86 & $1.765-1.913$ & 0.031 & 1.846 & $1.713-1.917$ & 0.013 & 1.89 & $1.694-2.132$ & 0.027 \\
\hline $\mathrm{HDL}$ & 0.25 & $0.086-0.541$ & 0.032 & 0.231 & $0.069-0.182$ & 0.021 & 0.516 & $0.285-0.930$ & 0.012 \\
\hline LDL & 0.985 & $0.897-1.153$ & 0.068 & 0.924 & $0.897-1.358$ & 0.21 & 0.427 & $0.203-0.947$ & 0.135 \\
\hline
\end{tabular}


Table 5 Multiple logistic regression analysis for CAD patients and control subjects of Uygur population(rs12441817)

\begin{tabular}{|c|c|c|c|c|c|c|c|c|c|}
\hline & \multicolumn{3}{|c|}{ Total } & \multicolumn{3}{|c|}{ Men } & \multicolumn{3}{|c|}{ Women } \\
\hline & OR & $95 \% \mathrm{Cl}$ & $\mathbf{P}$ & OR & $95 \% \mathrm{Cl}$ & $\mathbf{P}$ & OR & $95 \% \mathrm{Cl}$ & $\mathbf{P}$ \\
\hline Dominant model ( $T$ vs $C T+C C$ ) & 0.253 & $0.231-0.546$ & 0.016 & 0.241 & $0.132-0.478$ & 0.002 & 0.723 & $0.273-1.523$ & 0.362 \\
\hline Smoking & 9.152 & $6.278-13.373$ & $<0.001$ & 11.286 & $6.325-19.178$ & $<0.001$ & 0.471 & $0.357-1.932$ & 0.378 \\
\hline $\mathrm{EH}$ & 7.435 & $3.835-10.637$ & $<0.001$ & 9.513 & $4.631-20.278$ & $<0.001$ & 5.158 & $1.532-9.259$ & $<0.001$ \\
\hline DM & 1.338 & $0.754-2.269$ & 0.376 & 1.723 & $0.785-3.832$ & 0.173 & 0.632 & $0.209-2.371$ & 0.952 \\
\hline Glu & 1.432 & $1.341-1.573$ & $<0.001$ & 1.409 & $1.283-1.672$ & $<0.001$ & 1.538 & $1.327-1.849$ & $<0.001$ \\
\hline TG & 1.475 & $1.348-1.721$ & 0.032 & 1.469 & $1.258-1.732$ & 0.016 & 1.727 & $1.486-2.327$ & 0.011 \\
\hline TC & 1.532 & $1.467-1.853$ & 0.012 & 1.563 & $1.427-1.725$ & 0.028 & 1.542 & $1.329-1.935$ & 0.045 \\
\hline $\mathrm{HDL}$ & 0.305 & $0.132-0.529$ & 0.041 & 0.327 & $0.235-0.473$ & 0.035 & 0.273 & $0.075-0.683$ & 0.037 \\
\hline LDL & 0.653 & $0.532-1.243$ & 0.072 & 0.736 & $0.658-1.235$ & 0.237 & 0.639 & $0.347-0.953$ & 0.375 \\
\hline
\end{tabular}

CAD, coronary artery disease; EH, essential hypertension; DM, diabetes mellitus; Glu, glucose; TG, triglyceride; TC, total cholesterol; HDL, high-density lipoprotein; LDL, Low-density lipoprotein.

Association between CYP1A1 and CAD was different on account of polymorphisms of the CYP1A1 gene, Ethnic differences, environmental factors. CYP1A1 might cause CAD in some smokers or non-smokers. Their mechanisms of CYP1A1 were different. CYP1A1 metabolites arachidonic acid to 20-HETE, EETs, which cause CAD, in nonsmoking.

In this study, we hypothesized that variability in the CYP1A1 gene might affect the risk of CAD through CYP1A1 as an arachidonic acid epoxygenase enzyme in nonsmoking. We genotyped four SNPs of this gene in the Uygur and Han of China and assessed the association between CYP1A1 polymorphisms and CAD using a case-control analysis.

A significant difference was observed in the genotypic distribution of SNP1 (rs4886605) between the patients with $\mathrm{CAD}$ and controls within the full Uygur sample. When men and women were analyzed separately, the $\mathrm{T}$ allele frequency of rs4886605 was higher among men with CAD than their control counterparts. No such differences were observed among women. Thus, the risk of CAD is increased with regard to the $\mathrm{T}$ allele of rs 4886605 in men. The dominant model (CC vs CT + TT) was significantly lower among patients with CAD than controls for all participants and men; furthermore, this difference was retained after adjusting for Glu, LDL-C, EH, DM, and smoking (Table 4) using multiple logistic regression analyses. This finding indicated that the risk of CAD was decreased with the presence of the CC genotype of rs 4886605 in Uygur men. In the full Uygur sample, a significant difference was observed in the genotypic distribution of SNP2 (rs12441817) between patients with CAD and controls. When men and women were analyzed separately, the $\mathrm{C}$ allele of rs12441817 was more frequency in men with CAD than their control counterparts. No such differences were observed among women. Thus, the risk of CAD was increased with the appearance of the $C$ allele in rs12441817 among men. The dominant model (TT vs $\mathrm{CC}+\mathrm{CT}$ ) was significantly lower among patients with CAD than controls for all participants and men; furthermore, this difference remained significant after adjusting for Glu, LDL-C, EH, $\mathrm{DM}$, and smoking (Table 5). This finding indicates that the risk of CAD was decreased with the presence of the TT genotype in rs12441817 among Uygur men.

We found associations between CAD and the CYP1A1 SNPs rs4886605 and rs12441817 among Uygur men. This result might be caused by two reasons. First, it might be because of sex hormones; for instance, estrogen protects against oxidative stress and is vasoprotective [39-41]. The vascular protective effects of estradiol are metabolized into 2-hydroxyestradiol (2HE) by CYP1A1, and 2HE is converted into 2-methoxyestradiol (2ME) by catechol-Omethyl transferase. 2ME is antimitogenic, anti-angiogenic and pro-apoptotic [42]. Second, it might be because of less female samples. However, patients with CAD and controls participants in the Han group did not significantly differ with regard to the distributions of the rs4886605 and rs12441817 genotypes, the dominant model, the recessive model, or allele frequency $(\mathrm{P}>0.05)$. This genetic difference associated with coronary heart disease might differ across ethnic groups because of race,diet, or lifestyle.

Although SNP4 (rs1048943) was observed in the exon 7 region of the CYP1A1 gene and the polymorphisms caused a loss of transcription factor binding at site $\mathrm{Sp7}$, the synthesis of 20-HETE and EETs was reduced. Chih-Ching Yeh [38] showed that rs1048943 was independently associated with a decreased risk of coronary artery disease in nonsmoker $(\mathrm{OR}=0.32,95 \% \mathrm{CIs}=0.15-0.70)$ in the Taiwanese population, But they didn't find the association between rs1048943 and CAD in smoker. This study further indicated some polymorphisms of CYP1A1 can cause CAD through metabolism of 20-HETE and EETs excluding the risk factors like smoking in different ethnic groups. Our study was inconsistent with that study, showing no significant association between rs1048943 and CAD among nonsmoking participants in the Uygur and Han of china. 
Differences in populations such as race, geographical and environment factors, might explain these results between Chinese and Taiwanese population.

Few studies have focused on the association between SNP3 (rs4646422) in the exon 2 and CAD. We did not find significant differences between the patients with $\mathrm{CAD}$ and controls with regard to the distributions of the rs4646422 genotypes, the dominant model, the recessive model, or allele frequency among the Han and Uygur samples.

Our study has several limitations. On one hand, the present study analyzed only a small sample data, More studies will need to incorporate a large samplesize for confirming the association. On the other hand, further studies will need to be undertaken in order to clarify the underlying molecular mechanism that polymorphism of CYP1A1 gene was associated with CAD .

\section{Conclusions}

In conclusion, we found that rs4886605 and rs 12441817 might be two novel polymorphisms of the CYP1A1 gene associated with CAD in the Uygur population in China. The CC genotype of rs4886605 and the TT genotype of rs12441817 in the CYP1A1 gene might be protective genetic markers of CAD, whereas the $\mathrm{T}$ allele of rs4886605 and the $\mathrm{C}$ allele of rs12441817 might be genetic risk markers of $\mathrm{CAD}$ in the Uygur population in China.

\section{Competing interests}

The authors declare that they have no competing interests.

\section{Authors' contributions}

Conceived and designed the experiments: JG-Z, XX, Y-TM; Performed the experiments: JG-Z, XX, Y-NY, SP, DLR-AD, Analyzed the data: JG-Z, FL, Contributed reagents/ materials/ analysis tools: B-DC; Wrote the paper: JG-Z, Y-TM. All authors read and approved the final manuscript.

\section{Acknowledgements}

This work was supported financially by the Great Technology Special Item Foundation of Xinjiang Uygur Autonomous Region (201233138).

\section{Author details}

'Department of Cardiology, First Affiliated Hospital of Xinjiang Medical University, Urumqi 830054, People's Republic of China. ${ }^{2}$ Xinjiang Key Laboratory of Cardiovascular Disease Research, Urumqi 830054, People's Republic of China.

Received: 10 July 2014 Accepted: 2 September 2014

Published: 5 September 2014

\section{References}

1. Zordoky BNM, El-Kadi AOS: Effect of cytochrome P450 polymorphism on arachidonic acid metabolism and their impact on cardiovascular diseases. Pharmacol Ther 2010, 125:446-463.

2. Aboutabl ME, El-Kadi AOS: Constitutive expression and inducibility of CYP1A1 in the h9c2 rat cardiomyoblast cells. Toxicol In Vitro 2007 21(8):1686-1691.

3. Frazier L, Johnson RL, Sparks E: Genomics and cardiovascular disease. J Nurs Scholarsh 2005, 37(4):315-321.

4. Nordlie MA, Wold LE, Kloner RA: Genetic contributors toward increased risk for ischemic heart disease. J Mol Cell Cardiol 2005, 39(4):667-679.
5. Winkelmann BR, Hager J: Genetic variation in coronary heart disease and myocardial infarction: methodological overview and clinical evidence. Pharmacogenomics 2000, 1(1):73-94.

6. Niwa T, Murayama N, Yamazaki H: Oxidation of endobiotics mediated by xenobiotic-metabolizing forms of human cytochrome P450. Curr Drug Metab 2009, 10(7):700-712.

7. Elbekai RH, El-Kadi AO: Cytochrome P450 enzymes: central players in cardiovascular health and disease. Pharmacol Ther 2006, 112(2):564-587.

8. Zordoky BN, El-Kadi AO: Modulation of cardiac and hepatic cytochrome P450 enzymes during heart failure. Curr Drug Metab 2008, 9(2):122-128.

9. Wang XL, Greco M, Sim AS, Duarte N, Wang J, Wilcken DE: Effect of CYP1A1 Mspl polymorphism on cigarette smoking related coronary artery disease and diabetes. Atherosclerosis 2002, 162(2):391-397.

10. Cornelis MC, El-Sohemy A, Campos H: Genetic polymorphism of CYP1A2 increases the risk of myocardial infarction. J Med Genet 2004, 41(10):758-762.

11. Yasar U, Bennet AM, Eliasson E, Lundqren S, Wiman B, De Faire U, Rane A: Allelic variants of cytochromes $\mathrm{P} 4502 \mathrm{C}$ modify the risk for acute myocardial infarction. Pharmacogenetics 2003, 13(12):715-720.

12. Funk M, Endler G, Freitag R, Wojta J, Huber K, Mannhalter C, SunderPlassmann R: CYP2C9*2 and CYP2C9*3 alleles confer a lower risk for myocardial infarction. Clin Chem 2004, 50(12):2395-2398.

13. Spiecker M, Darius H, Hankeln T, Soufi M, Sattler AM, Schaefer JR, Node K, Börgel J, Mügge A, Lindpaintner K, Huesing A, Maisch B, Zeldin DC, Liao JK: Risk of coronary artery disease associated with polymorphism of the cytochrome P450 epoxygenase CYP2J2. Circulation 2004, 110(15):2132-2136.

14. Nakayama T, Soma M, Saito S, Honye J, Yajima J, Rahmutula D, Kaneko Y, Sato M, Uwabo J, Aoi N, Kosuge K, Kunimoto M, Kanmatsuse K, Kokubun S: Association of a novel single nucleotide polymorphism of the prostacyclin synthase gene with myocardial infarction. Am Heart J 2002, 143(5):797-801.

15. Hengstenberg C, Holmer SR, Mayer B, Löwel H, Engel S, Hense HW, Riegger GA, Schunkert H: Evaluation of the aldosterone synthase (CYP11B2) gene polymorphism in patients with myocardial infarction. Hypertension 2000, 35(3):704-709.

16. Letonja M, Peterlin B, Bregar D, Petrovic D: Are the T/C polymorphism of the CYP17 gene and the tetranucleotide repeat (TTA) polymorphism of the CYP19 gene genetic markers for premature coronary artery disease in Caucasians? Folia Biol (Praha) 2005, 51:76-81.

17. Choudhary D, Jansson I, Stoilov I, Sarfarazi M, Schenkman JB: Metabolism of retinoids and arachidonic acid by human and mouse cytochrome P450 1B1. Drug Metab Dispos 2004, 32(8):840-847.

18. Schwarz D, Kisselev P, Ericksen SS, Szklarz GD, Chernogolov A, Honeck H, Schunck WH, Roots I: Arachidonic and eicosapentaenoic acid metabolism by human CYP1A1: highly stereoselective formation of 17(r), 18(s)epoxyeicosatetraenoic acid. Biochem Pharmacol 2004, 67(8):1445-1457.

19. Rifkind AB: CYP1A in TCDD toxicity and in physiology-with particular reference to CYP dependent arachidonic acid metabolism and other endogenous substrates. Drug Metab Rev 2006, 38(1-2):291-335.

20. Fleming I: Cytochrome P-450 under pressure: more evidence for a link between 20-hydroxyeicosatetraenoic acid and hypertension. Circulation 2005, 111(1):5-7.

21. Manfredi S, Federici C, Picano E, Botto N, Rizza A, Andreassi MG: GSTM1, GST11 and CYP1A1 detoxification gene polymorphisms and susceptibility to smoking-related coronary artery disease: a case-only study. Mutat Res 2007, 621(1-2):106-112.

22. Franchi E, Marino P, Biondi-Zoccai GG: Transradial versus transfemoral approach for percutaneous coronary procedures. Curr Cardiol Rep 2009, 11(5):391-397.

23. Vazquez B, Rios A, Escalante B: Arachidonic acid metabolism modulates vasopressin-induced renal vasoconstriction. Life Sci 1995, 56(18):1455-1466.

24. Chu ZM, Croft KD, Kingsbury DA, Falck JR, Reddy KM, Beilin LJ: Cytochrome P450 metabolites of arachidonic acid may be important mediators in angiotensin Il-induced vasoconstriction in the rat mesentery in vivo. Clin Sci (Lond) 2000, 98(3):277-282.

25. Imig JD, Pham BT, LeBlanc EA, Reddy KM, Falck JR, Inscho EW: Cytochrome P450 and cyclooxygenase metabolites contribute to the endothelin-1 afferent arteriolar vasoconstrictor and calcium responses. Hypertension 2000, 35(1 pt 2):307-312. 
26. Fu Z, Nakayama T, Sato N, Izumi Y, Kasamaki Y, Shindo A, Ohta M, Soma M Aoi N, Sato M, Matsumoto K, Ozawa Y, Ma Y: Haplotype-based case study of human CYP4A11 gene and cerebral infarction in Japanese subject. Endocrine 2008, 33(2):215-222.

27. Uddin MR, Muthalif MM, Karzoun NA, Benter IF, Malik KU: Cytochrome P-450 metabolites mediate norepinephrine-induced mitogenic signaling. Hypertension 1998, 31(2):242-247.

28. Muthalif MM, Karzoun NA, Gaber L, Khandekar Z, Benter IF, Saeed AE, Parmentier JH, Estes A, Malik KU: Angiotensin Il-induced hypertension: contribution of Ras GTPase/Mitogen-activated protein kinase and cytochrome P450 metabolites. Hypertension 2000, 36(4):604-609.

29. Schwartzman ML, Abraham NG, Masferrer J, Dunn MW, McGiff JC: Cytochrome P450 dependent metabolism of arachidonic acid in bovine corneal epithelium. Biochem Biophys Res Commun 1985, 132(1):343-351.

30. Quigley R, Baum M, Reddy KM, Griener JC, Falck JR: Effects of 20-HETE and 19(S)-HETE on rabbit proximal straight tubule volume transport. Am J Physiol Renal Physiol 2000, 278(6):949-953.

31. McGiff JC, Quilley J: 20-Hydroxyeicosatetraenoic acid and epoxyeicosatrie noic acids and blood pressure. Curr Opin Nephrol Hypertens 2001 10(2):231-237.

32. Gross GJ, Falck JR, Gross ER, Isbell M, Moore J, Nithipatikom K: Cytochrome P450 and arachidonic acid metabolites: role in myocardial ischemia/ reperfusion injury revisited. Cardiovasc Res 2005, 68(1):18-25.

33. Nithipatikom K, Gross ER, Endsley MP, Moore JM, Isbell MA, Falck JR, Campbell WB, Gross GJ: Inhibition of cytochrome P450omegahydroxylase: a novel endogenous cardioprotective pathway. Circ Res 2004, 95(8):e65-e71.

34. Hill E, Fitzpatrick F, Murphy RC: Biological activity and metabolism of 20-hydroxyeicosatetraenoic acid in the human platelet. Br J Pharmacol 1992, 106(2):267-274.

35. Spiecker M, Liao JK: Cytochrome P450 epoxygenase CYP2J2 and the risk of coronary artery disease. Trends Cardiovasc Med 2006, 16:204-208 [PubMed].

36. Kawajiri K, Watanabe J, Hayashi S: Identification of allelic variants of the human CYP1A1 gene. Methods Enzymol 1996, 272:226-232.

37. Nerurkar PV, Okinaka L, Aoki C, Seifried A, Lum-Jones A, Wilkens LR, Le Marchand L: CYP1A1, GSTM1, and GSTP1 genetic polymorphisms and urinary 1-hydroxypyrene excretion in non-occupationally exposed individuals. Cancer Epidemiol Biomarkers Prev 2000, 9(10):1119-1122.

38. Yeh C-C, Sung F-C, Kuo L-T, Hsu WP, Chu HY: Polymorphisms of cytochrome P450 1A1, cigarette smoking and risk of coronary artery disease. Mutat Res 2009, 667:77-81.

39. Haschke-Becher E, Kirchheiner J, Trummer O, Grünbacher G, Kainz A: Impact of CYP2C8 and 2C9 polymorphisms on coronary artery disease and myocardial infarction in the LURIC cohort. Pharmacogenomics 2010, 11:1359-1365 [PubMed].

40. Kondo T, Hirose M, Kageyama K: Roles of oxidative stress and redox regulation in atherosclerosis. J Atheroscler Thromb 2009, 16:532-538 [PubMed].

41. Kaur-Knudsen D, Bojesen SE, Nordestgaard BG: Common polymorphisms in CYP2C9, sub-clinical atherosclerosis and risk of ischemic vascular disease in 52000 individuals. Pharmacogenomics J 2009, 9:327-332 [PubMed].

42. Tofovic SP: Estrogens and development of pulmonary hypertension Interaction of estradiol metabolism and pulmonary vascular disease. J Cardiovasc Pharmacol 2010, 56(6):696-708.

doi:10.1186/1476-511X-13-145

Cite this article as: Zou et al:: The association between CYP1A1 genetic polymorphisms and coronary artery disease in the Uygur and Han of China. Lipids in Health and Disease 2014 13:145.

\section{Submit your next manuscript to BioMed Central and take full advantage of:}

- Convenient online submission

- Thorough peer review

- No space constraints or color figure charges

- Immediate publication on acceptance

- Inclusion in PubMed, CAS, Scopus and Google Scholar

- Research which is freely available for redistribution

Submit your manuscript at www.biomedcentral.com/submit
C Biomed Central 